\title{
Malnutrition, frailty and quality of life in older adults - a cross cultural study
}

\author{
Eirini Kelaiditi, Maria Teresa Vidal Vidal and Kyriaki Myrissa \\ St Mary's University Twickenham, London, United Kingdom
}

\section{Abstract}

Introduction: Life expectancy has considerably improved over the past century. Despite the population living longer, this greater number of life years has been associated with increases in chronic morbidity and mortality, including age-related debilitating conditions, such as frailty and malnutrition with profound implications both socially and economically on society. Given the increasing rates of population ageing, it is critical to ensure that functional ability, wellbeing and quality of life (QoL) are maintained and the factors involved in these processes are understood. This project aimed to compare the prevalence of frailty and malnutrition in community-dwelling older adults living in the UK and Spain; and identify potential determinants of malnutrition, frailty and QoL.

Materials and methods: A cross sectional study among 64 male and female community-dwelling older adults 65-92 years from the UK $(\mathrm{n}=33)$ and Spain $(\mathrm{n}=31)$ was conducted. Nutritional status, frailty and QoL were assessed by previously validated self-reported questionnaires: the Mini Nutritional Assessment Short Form (MNA-SF), the FiND (Frail Non-Disabled questionnaire) and the SHARE (Study of Health Ageing and Retirement in Europe) version of the CASP-12. Multiple and/or logistic regression models were applied to assess factors that predict malnutrition, QoL and risk of frailty.

Results: In total, mean age of participants was $77 \pm 8$ years and mean Body Mass Index (BMI) was $26.06 \pm 4.78 \mathrm{~kg} / \mathrm{m}^{2}$. Mean MNA-SF score was $12.70 \pm 1.92$, and mean QoL was $40.36 \pm 5.35$. The overall prevalence of malnutrition and frailty was $18.8 \%$ and $23.4 \%$ respectively. Participants in the UK were more likely to be malnourished than those in Spain $(24.2 \%$ vs $12.9 \%)$, although not significantly $(\mathrm{p}=0.245)$, and the prevalence of frailty was higher in the UK $(36.4 \%$ vs $9.7 \%)(p=0.012)$. BMI, being frail and income were significant predictors of malnutrition $(\mathrm{p}<0.05)$. Higher QoL was a significant protective factor for frailty risk $($ Odds Ratio 0.728; 95\%CI 0.59-0.90; $\mathrm{p}=0.004)$. Age, income and being frail were significant predictors of QoL $(\mathrm{p}<0.05)$.

Discussion: To our knowledge this is the first cross-cultural study that explored associations between malnutrition, frailty and QoL, using validated questionnaires in both languages. Despite the small sample size, it was revealed that malnutrition and frailty are conditions that can be identified in community dwelling older adults. Although participants self-reported a good QoL, frailty was found to be a strong predictor of QoL. Implementing culturally suited strategies to detect malnutrition and frailty status and prevent further decline would be useful to improve the QoL of older adults in these countries.

\section{Conflict of Interest}

There is no conflict of interest 